\title{
The Interactive Shared Educational Environment: User Interface, System Architecture and Field Study
}

\author{
Xiangming Mu, Gary Marchionini, and Amy Pattee \\ School of Information and Library Science \\ University of North Carnolina at Chapel Hill \\ \{mux,march\}@ils.unc.edu,patta@email.unc.edu
}

\begin{abstract}
The user interface and system architecture of a novel Interactive Shared Educational Environment (ISEE) are presented. Based on a lightweight infrastructure, ISEE enables relatively low bandwidth network users to share videos as well as text messages. Smartlink is a new concept introduced in this paper. Individual information presentation components, like the video player and text chat room, are "smartly" linked together through video timestamps and hyperlinks. A field study related to children book selections using ISEE was conducted. The results indicated that the combination of three information presentation components, including video player with storyboard, shared browser, and text chat room, provided an effective and more comfortable collaboration and learning environment for the given tasks than text reviews or text chat alone or in combination. The video player was the most preferred information component. Text comments in the chat room that did not synchronize with the video content distracted some participants due to limited cognitive capacity. Using smartlink to synchronize various information components or "channels" is our attempt to reduce the user's working memory load in information enriched distance learning environments made possible by digital libraries.
\end{abstract}

\section{Introduction}

Distance learning separates learners from instructors in space and/or time. Various web-based communication technologies have been adopted in distance learning to connect instructors and learners. Email, hypertext web pages, online forums, email-lists, and Bulletin Board Systems (BBS) are examples of some widely used technologies in current distance learning systems, like the Blackboard system [24]. The increase network bandwidth and computer technology also makes multimedia content such as video and real-time communication and interactive $\mathrm{TV}$, to be incorporated into distance learning. Digital libraries are especially helpful in providing rich content for distance education.
Video digital libraries provide especially good potential for distance learning. Video is able to vividly present complex, abstract concepts and microscopic intricacies; to stimulate thought and emotions through evocative drama or documentary; and to capture and replay events $[3,10]$. The advance of digital libraries and "open" video projects such as the Internet Archive project (http://www.archive.org) and the Open-video project (www.open-video.org), provide an increasing collection of videos that can be directly accessed through the web by remote learners. With these advances, how to take advantage of video to enhance distance learning becomes a new challenge.

Even with the adoption of advanced compression and encoding technology, like Moving Picture Expert Group (MPEG), video is still an expensive medium with respect to the amount of information transferred over the Internet. Thus another challenge in the distance-learning domain is how to enable low bandwidth network users to get benefits from the new multimedia distance learning tools.

As a tradeoff, decreasing the video quality is routinely utilized to guarantee video continuity for low bandwidth connections. However, this approach constrains the applications of the video and limits the benefits provided by high quality videos. Instruction that focuses on details and requires high resolution images will fail under such a environment.

Streaming, a video delivery format using "push" technology that is similar to sending TV programs, is often adopted to broadcast real-time videos over the Internet. Various qualities of the same video are usually provided as different channels for users to select in accordance with their particular network connection speeds. However, the drawback is that streaming is a oneway technology and few interaction functions are supported. For example, users are not allowed to freely roll back the video player to re-view the past content or forward the video to preview.

Based on a new text-based communication protocol, the Interactive Shared Educational Environment (ISEE) is a novel multimedia distance learning and collaboration tool that is specifically designed for low bandwidth network users. Real-time video sharing among learners and instructors, as well as video navigation functions is 
supported in the ISEE. By seamlessly integrating four information presentation components, including a high quality pre-packaged digital video player, a video storyboard, a shared web browser for video scripts and web pages, and a real-time text chat room, ISEE provides a rich collaborative learning environment for distributed users. Timestamps of the video are utilized to "synchronize" the contents of each individual information component, a concept we refer to as "smartlink". Immense web information is also accessible and sharable with the help of the built-in web browser enabled component.

This paper is organized as follows: after a brief introduction to the related research, the ISEE user interface and its functionalities are introduced. In the following system architecture section, the concept of "smartlink" is proposed and discussed. Finally the results of a field study using ISEE are presented.

\section{Related work}

Two temporal modes of distance learning are synchronous and asynchronous. In terms of the direction of delivering instruction, distance learning also can be classified into one-way "push" instruction and two-way interactive instruction. Online chat, instant messaging, audio/video conferencing, shared real-time applications, computer supported cooperative work (CSCW) tools, and interactive television (ITV) are examples of two-way synchronized distance learning (Table 1).

New tools have been developed using more than one medium to provide a more effective or customized learning environment. MediaSite Live [15] from Sonic Foundary is a web-based application built on ASP technology that supports one-way video streaming, which is synchronized with associated PowerPoint ${ }^{\mathrm{TM}}$ slides. Windows Media Player ${ }^{\mathrm{TM}}$ is used in MediaSite Live to present the streaming video. A minimum bandwidth is required for the video playback $(240 * 180$ with $15 \mathrm{fps})$. No interaction or collaboration functions are supported.

Stanford University's Stanford Online program [19] adopts a similar approach. In addition to asynchronous streaming video, a synchronous videoconference system is also supported for the purpose of two-way interaction.

The Just-In-Time Lecture Project (JIT) developed at Carnegie Mellon University embedded high resolution slides directly into the video to guarantee "synchronization" between slides and video. E-mail is used to link instructors and learners [12].

The BMRC Lecture Browser [2], developed at the UC Berkeley Multimedia Research Center also uses streaming technology. A visual segmented-video bar is added to facilitate video browsing.
Table 1: Media Used in Distance Learning

\begin{tabular}{|c|l|l|}
\hline Modes & \multicolumn{1}{|c|}{ One-way } & \multicolumn{1}{|c|}{ Two-way } \\
\hline \multirow{3}{*}{ Asyn } & $\begin{array}{l}\text { Hypertext Webpage, } \\
\text { Video streaming, } \\
\text { Video on demand, } \\
\text { CD-R, Video tapes, } \\
\text { etc. }\end{array}$ & $\begin{array}{l}\text { Mail, Email, BBS, Fax } \\
\text { online Forum, Email } \\
\text { list, etc. }\end{array}$ \\
\hline \multirow{3}{*}{ Syn } & $\begin{array}{l}\text { Radio/TV } \\
\text { broadcasting, Real- } \\
\text { time Streaming, etc. }\end{array}$ & $\begin{array}{l}\text { Instant messaging, } \\
\text { Text Chat, Telephone, } \\
\text { MUD/MOO*, } \\
\text { Audioconferening, } \\
\text { Videoconference, } \\
\end{array}$ \\
& & $\begin{array}{l}\text { Interactive TV, etc. } \\
\text { *Multi-User Domains/ MUD, Object Oriented }\end{array}$ \\
\hline
\end{tabular}

eClass [8] from Georgia Tech (formerly called Class 2000) captures class activities using a video camera and an electronic whiteboard, which are integrated together along a timeline. Slides generated from the electronic whiteboard are linked to the timeline for quick reference. One unique contribution of eClass involves creation and use of hyperlinks attached to each slide to connect to the related video segments. HTML and JavaScript are utilized to implement the eClass environment.

Studies $[9,17,18,20]$ have indicated that students who collaboratively watched video outperformed students who attended live lectures in the traditional classroom as well as students who watched live streaming lectures individually in both situations with or without tutors (mediators). The success in these studies is attributed to the encouragement of collaboration. However, most of the distance-learning systems fail to integrate direct supports for collaboration. External communication tools, such as email or online forums, are usually used for information exchanging or sharing.

Videoconferencing systems were chosen by some education systems to facilitate collaboration across users $[6,7,13,16,22]$ due to their direct support for two-way interaction. Research demonstrated, however, that little benefit is gained from including talking-head video or videoconferencing [4, 5] for communication in the educational domain. Special requirements for video capture and video encoding/decoding equipment limited the range of their applications. In addition, high speed network connectivity is usually necessary for smooth video presentation.

Realizing the importance of two-way communication between the instructor and remote learners, TELEP, developed by Microsoft, added a dialog chat to the oneway video/slide system [11]. Remote users are able to send questions to the presenter directly, and other users can also join the discussion by replying to the question or issuing comments. However, real-time multicast technology used in the TELEP for the video/audio streaming means users not only cannot rollback or 
navigate the video while watching, but also had to have a multicast enabled network connection, which is not common for most dialup network users.

ISEE is a real-time collaborative multimedia distance learning tool suitable for heterogeneous network connections. ISEE is distinguished by the following characteristics:

- ISEE supports real-time text chat, video playback, video navigation with a storyboard, video synchronization across users, hypertext browsing, web information access, and web site URL sharing. All these functions are integrated together to form a cohesive learning environment.

- Text chat messages, video scripts and video are linked "smartly". With a single click on the timestamp associated with the message presented in the chat window, a user can automatically synchronize or "align" his/her video to the point in the video playing when the sender wrote the message.

- ISEE can be applied to heterogeneous network connections, including slow dialup networks when video content is stored locally.

- ISEE is operating system independent.

\section{User interface}

A desktop Graphic User Interface (GUI) was designed in ISEE to accommodate various learning and collaboration components. (Fig.1). One of the benefits of this style is that users can customize their learning environment by re-arranging the number, positions, and size of each component in accordance with their personal learning styles. This is particularly useful when multiple communication and learning components are used simultaneously. Another advantage is that it can take full advantage of screen real estate when the users' screen resolutions are high. For example, students can enlarge the relative size of a video player in a high-resolution $(1600 * 1248)$ monitor to get a better video display rather than using the default size, which is designed for a screen resolution of $1024 * 768$.

Figure 1 presents a sample screenshot of the ISEE user interface. On the top of the interface is a menu bar that contains a series of buttons that can open corresponding sub-panels in the desktop panel beneath: an Interactive Chat Room (ICR) sub-panel, a user profile sub-panel, a video player sub-panel, a storyboard sub-panel, and a Shared Web Browser (SWB) sub-panel. Buttons for selecting a video, logging in, marking time stamps, and other functions are also available.

In Figure 1, the top right of the desktop panel displays the user profile sub-panel, which lists users currently logged in. Beneath it is the Interactive Chat Room (ICR) sub-panel that allows users to share text chat. The sender's name, timestamp of the video, and the message are displayed. The SWB is located on the left side of the desktop panel to display hypertext documents, which can be shared among users. The video player and storyboard sub-panels are in the middle of the ISEE interface in default mode. Thumbnails of the video frames are displayed on the storyboard for video content navigation. Thumbnails are generated using our procedures developed for the Open Video Digital Library. There is a slider bar just beneath the video player. Users are able to drag the bar to change the current playback point or use it to preview the content of the video quickly.

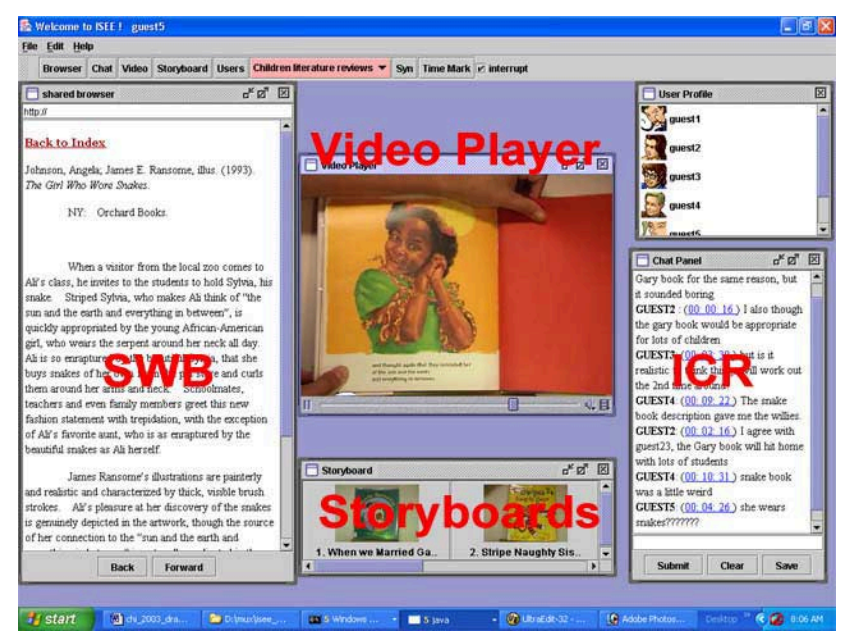

Figure 1: ISEE User Interface

The four learning and communication components are not only juxtaposed together in appearance, but also coherently connected with each other through a mechanism called "smartlink".

\section{Smartlinks}

Each of the learning and communication components in the ISEE, including ICR, SWB, video player, and storyboards, are "smartly" linked through the video timestamps or hypertext URLs. We refer to this feature as "smartlink". Table 2 lists the current available smartlinks.

In ISEE, the Interactive Chat Room (ICR) is different from the generic online text chat tools in terms of supporting interaction between users via messages. Each message sent by a user contains not only the sender's username and the message content, but also the sender's video timestamp representing the point when the message was sent (Fig. 2). A single click on the timestamp by a particular user immediately updates that user's local video player to the point in the video corresponding to the timestamp. Thus any user who is interested in a particular 
message is able to share both the text message and the video context associated with the message. Such a mechanism brings convenience to users who want to discuss topics that are tightly associated with the video context. For example, when discussing a video picture book, a message such as "look at the little thing sitting on the nose of the bear, is it a mouse?" makes no sense to another user if he/she cannot share the same scene.

Table 2: Smartlinks Between ISEE Components

\begin{tabular}{|l|l|c|c|c|}
\hline & ICR & SWB & Storyboard & $\begin{array}{l}\text { Video } \\
\text { Player }\end{array}$ \\
\hline ICR & & yes & no & yes \\
\hline SWB & yes & & no & yes \\
\hline Storyboard & no & no & & yes \\
\hline Video Player & yes & yes & yes & \\
\hline
\end{tabular}

ICR also supports hypertext links just like a generic web browser. The hyperlink is formed automatically when the message contains "http", "HTTP", "www" or "WWW". A single left click on such links immediately updates the display content in the SWB with the corresponding web page. With the help of this feature, users are able to take advantage of the immense information resources available in the Internet and share directly within the ISEE environment (Fig.2).

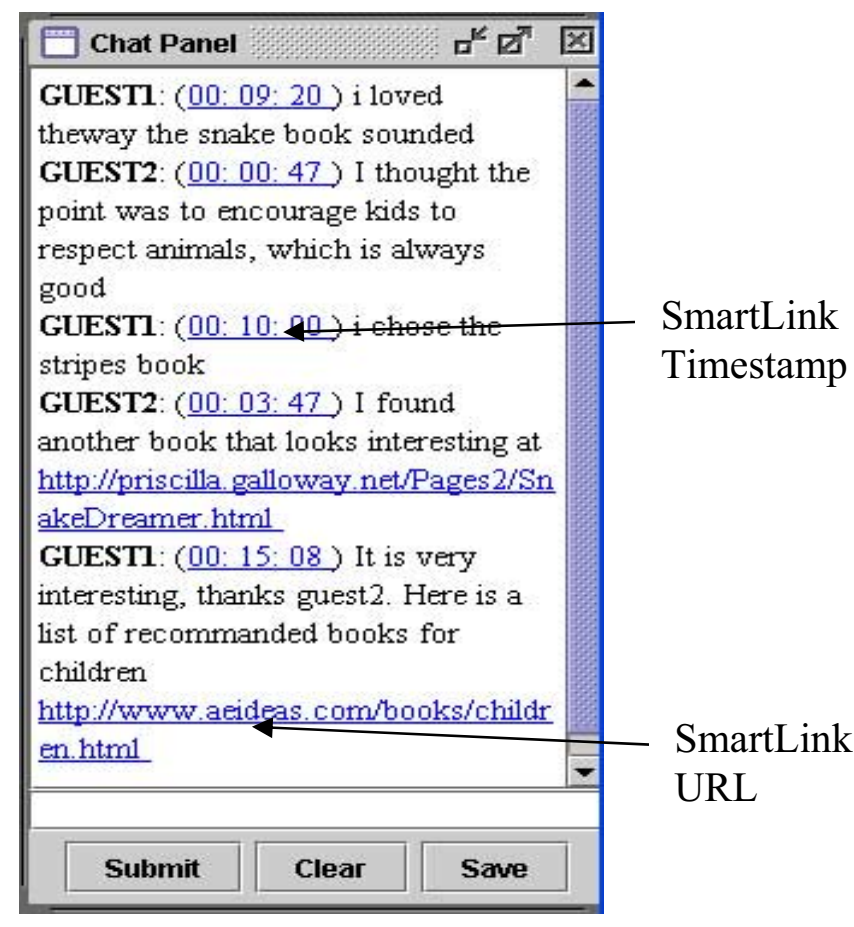

Figure 2: Chat Room with Smartlink Support

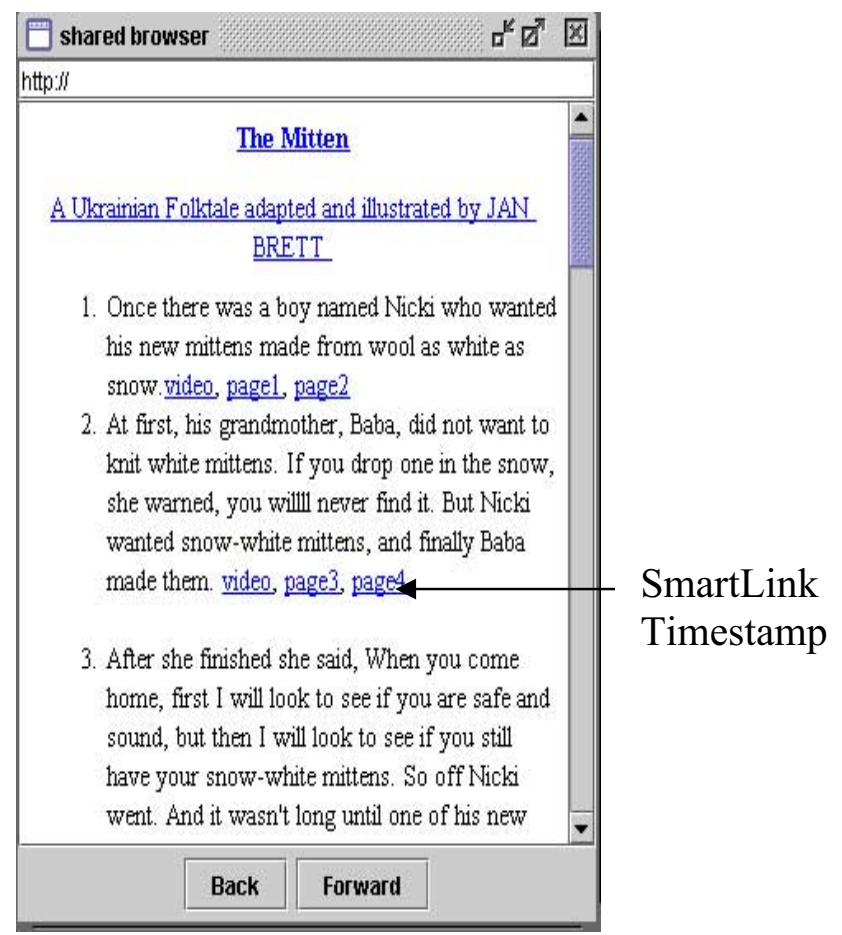

Figure 3: Shared Web Browser with Smartlink Support

The Shared Web Browser (SWB) is a built-in web browser we developed using Java. The SWB enables users to share hypertext documentation without leaving the ISEE. One way we have used the SWB in classroom demonstrations is to display session notes and assignments. There are three parts in the SWB panel: On the top is a text field that enables users to provide a URL. Beneath it is the hypertext area used for displaying the content of the URL. On the bottom there are two web page caching buttons that enable users to view the history of browsing. Individual users are able to use SWB to share hypertext documents. When a return key is pressed after a URL is given, not only that user's SWB is updated, other users logged in to the same session are also updated with the same web page referred to by the URL.

Smartlinking between the SWB and the video player is supported in the ISEE. Figure 3 illustrates a sample screenshot of the SWB component in which a transcript of the video generated from the children's book "The Mitten" is presented. Underlined texts in blue are smartlinks that contains the timestamps of the video player. A single left click on the text will immediately update the local video player to begin to playback from the corresponding segment.

\section{ISEE System}

\subsection{Overview}


Our practical goal is to design a new multimedia distance-learning environment based on the current computer and network technologies that enables distributed learners with typically modest network connections to get benefits from multimedia resources in digital libraries. Such an environment will allow instructors to develop instruction that takes advantage of video content. Thus, interactions with the video, such as pausing, stopping, and navigating, must be available. Instead of streaming video, we used pre-packaged video (either downloaded outside of class or collaborative study time, or provided on CDs mailed to remote students) to reduce delay and pre-caching time. Of course, a small number of users on an Internet-2 network could use ISEE with distributed video but such situations will remain the exception rather than the rule for some time for distance learning.

The environment itself will enable us to attack our larger goals of understanding how people make sense of video and more specifically how people manage multiple information channels while learning and working. Toward these ends, session logging is built into the ISEE to allow interactions at various levels of granularity to be captured for analysis.

Java was chosen as the development language to achieve interoperability across commonly used platforms. Java Shared Data Toolkits (JSDT) APIs were utilized for information sharing and group communication functions. Java Media Frame (JMF) APIs were used for developing the video related components, including the video player and video storyboards.

\subsection{System architecture}

Figure 4 illustrates the framework for the ISEE system. The Session Managing Service (SMS) is a daemon service that manages user interactions. Each user registers in the SMS by logging in. Text messages are shared across each registered user via SMS. A database that maps the user names with the Java objects is built in the SMS. Java sockets are used as the communication protocol across each client.

The logging module is a special "user" that logs selected activities within the session. Actions issued by users automatically trigger the "WriteLog" method built into each client. A message that contains the information about the action is sent by the "WriteLog" method to the Logging module, which records the received messages in the backend MySQL database

The MySQL database provides support for both the Login Check Module and the Logging Module. Only authorized users can join the session and share information. Actions and messages captured by the Logging Module are stored in the database for further analysis or system maintenance.

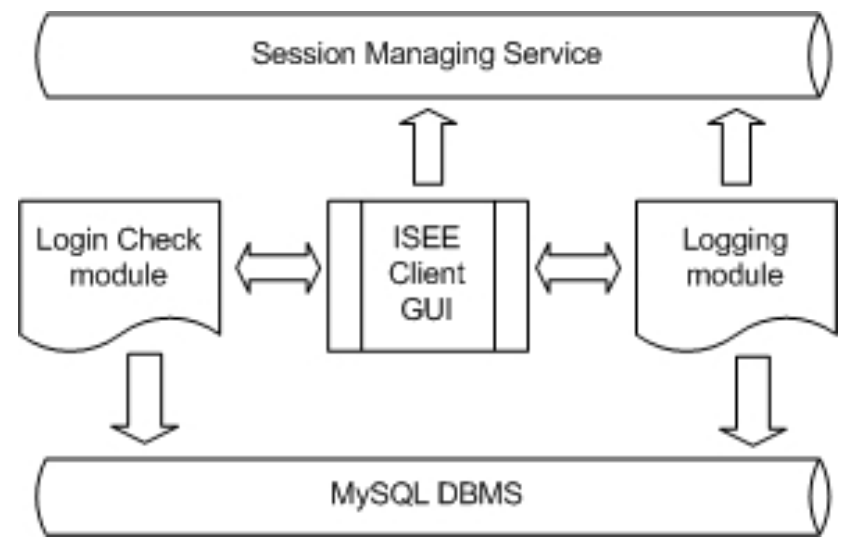

Figure 4: Framework for ISEE Architecture

\subsection{Flexibility}

A lightweight multimedia communication protocol has been developed for the ISEE to provide flexibility on heterogeneous network connections (Table 3).

Table 3: Communication Protocol in ISEE

\begin{tabular}{|c|c|}
\hline Tags & Functions \\
\hline$<$ Message $>\ldots</$ Message $>$ & Text message from users \\
\hline $\begin{array}{l}<\text { TextColor }>\ldots</ \text { TextColor } \\
>\end{array}$ & Text color from users \\
\hline$<$ Status $>\ldots</$ Status $>$ & $\begin{array}{l}\text { Status information from } \\
\text { users }\end{array}$ \\
\hline $\begin{array}{l}<\text { SynVideo }> \\
\ldots</ \text { SynVideo }>\end{array}$ & Timestamp from users \\
\hline$<$ URL $>\ldots</$ URL $>$ & URL from users \\
\hline$<$ VideoID $>\ldots<$ /VideoID $>$ & Video ID from users \\
\hline
\end{tabular}

In this protocol, text messages and video metadata, such as the timestamps of the video, are transmitted across clients for information sharing. The heavyweight video, in contrast, is pre-cached in each member user's local storage and is not transmitted. The collaboration is achieved through parsing the coded lightweight text metadata instead of the heavyweight video itself. In other words, the video metadata is shared instead of the video. To synchronize the video playback between two users, for an example, one user sends his/her local video timestamp to another user, instead of sending the video stream itself. As a result, even users with slow dialup connections are able to take advantage of the real-time multimedia collaborative environment.

\section{Field study}

\subsection{Instruction}


In order to evaluate the design and performance of the ISEE, user and field studies are needed. The initial version of ISEE was demonstrated at the Internet2 2002 Spring meeting in Virginia. At that demo, two users (one at the conference site and one in North Carolina) were logged in to mimic the real-time distance instruction between the instructor and a remote learner. Early in the Fall 2002 semester, the ISEE was used by one of the authors in a class of thirty students where several students participated at remote locations in the building using their laptops via a wireless network. In that case, performance dropped dramatically as more remote students joined the discussion. Also in that version of the ISEE, an additional multicast component beyond the ICR, SWB, and local video functions were used to broadcast the classroom activity. Based on that experience, the system infrastructure was rebuilt to solve the scalability problem and the multicast feature was dropped. The revised version of ISEE was tested in November, 2002 in a classroom field study.

\subsection{Participants and procedure}

Volunteers were recruited to participate in the field study from a population of students in the Children's Literature \& Related Materials course. Twenty-eight students participated in the study. Most were female (only two males) and they ranged in age from 20 to 45 . Eighteen participants were graduate students and all claimed that they used computers and the Internet on a daily basis. Two-thirds of the participants (19 of 28) never or occasionally had used interactive online chat. Most of the participants $(89 \%)$ had no past experience with video conferencing systems and about one-third (10 of 28) of the participants had taken an online class.

Participants were asked to assume that they were librarians and should order three children books for their library from five available selections. They were asked to read reviews of each book and to use ISEE to get peer opinions to help them make their decisions. The study was conducted in the computer lab with $10 \mathrm{Mb}$ fast Ethernet connected to each computer and ISEE preinstalled on each workstation along with the video files. Each participant was assigned a random user name from a pool ("user1" to "user31"). Although the decisionmaking class session was conducted in a single computer laboratory, each student worked at an individual workstation and wore headphones in phase three when the video was available. No talking was allowed during the entire study. Thus, the test session was a reasonable simulation of a distance learning setting.

There were three phases in the study: review, review + chat, and review + chat + video:
- Phase I: The SWB was used to browse text reviews of each book;

- Phase II: The ICR was used to share text messages with other group members; the SWB was still accessible in this phase.

- Phase III: Participants were asked to watch brief video readings for each book. Each page was shown as it was read. Storyboards were available for quick shifting between books. The SWB and ICR were available in this phase

Before the study, a brief tutorial (a few minutes) was given to let participants become familiar with the interface and functions of the ISEE system. After each phase, participants made or revised their book selections. Finally, a questionnaire with Likert scales assessing each of the components and various preferences and binary choices for preference and satisfaction, was provided to elicit reactions from participants on their experience using ISEE. The entire field test lasted about one hour.

\subsection{Results}

Results for basic performance and for specific features are discussed.

6.3.1. ISEE. The tested subjects found the ISEE easy to learn. Although the field test marked the first time any participant had used ISEE and only a brief tutorial was given, only three participants asked for help on how to use ISEE or its functions during the entire study. As user24 commented after the study, "It was amazingly easy to use". Additionally, ISEE was fairly stable and robust during the study. Only two participants re-started their ISEE environments.

6.3.2. Video, chat discussion, and text reviews. In this study, video was reported to be the most effective information channel in helping the participants make their final book selections. Only $3.6 \%$ of the participants selected the online chat and $7.1 \%$ selected the text review as most useful informant of their decision-making. The other $89 \%$ reported that the video was the most effective information resource. Participants felt more comfortable $(\mathrm{t}=4.42, \mathrm{df}=27, \mathrm{p}<0.001)$ using the preview + chat + video setting and found that this setting was more effective than the preview + chat setting $(\mathrm{t}=10.5, \mathrm{df}=27, \mathrm{p}<0.001)$. Most of the participants gave positive comments about the video. For examples: "The video was extremely helpful" (user12). "The videos were excellent! Great resources! This is a wonderful idea" (user17). "To see books with reviews helped immensely. (Video) changed my mind" (user19). In addition, even though we did not formally introduce the navigation functions associated 
with the video player, some participants tried them and indicated that they were helpful and convenient.

6.3.3. Smartlinks. As this was the first time for participants to use the ISEE user interface, the smartlink feature was not emphasized in the tutorial. However, most of the participants still used the storyboards for video navigation. Figure 5 shows the distribution of the 73 clicks on the video storyboards. The mean number of clicks per participant was 2.54 and the figure shows how usage was heavy early in phase 3 while participants explored the videos and then trailed off as they moved toward finalizing their selection decisions.

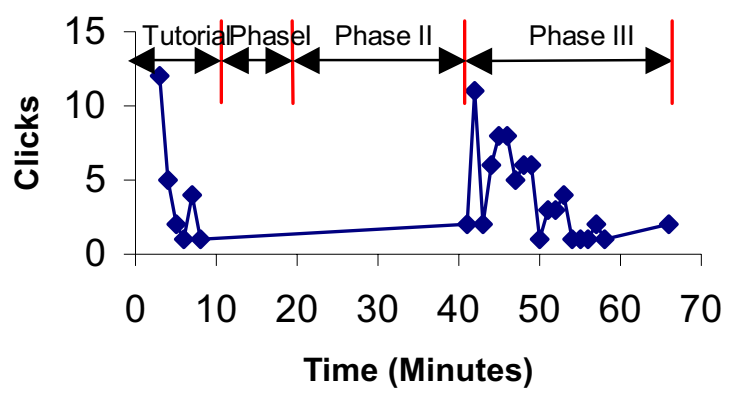

Figure 5. Storyboard Click Distribution Over Session

Five participants used the smartlink between the ICR and the video player. There were a total of twenty-five clicks on the linked timestamps. Table 4 lists the number of clicks on the ICR timestamps by each of the five users who used the smartlinks.

Table 4: ICR Timestamp Clicks

\begin{tabular}{|l|c|c|c|c|c|}
\hline User ID & User01 & User10 & User15 & User19 & User24 \\
\hline Clicks & 7 & 3 & 3 & 1 & 11 \\
\hline
\end{tabular}

User24 was the most active user in terms of using smartlinks. This suggests that she was very serious about the comments from others and used smartlinks in the ICR to share the same video context with the message sender. However, she remarked negatively on her chat room experience ("the chat rooms conversation got pretty silly") and she ranked the chat room as the least effective module in order of helpfulness. User10 gave a relatively positive remark about the smartlink: "I liked being able to chat while watching the videos and linking straight to the spot where another user was at when commenting". It is interesting to note that user25, who did not use the timestamp smartlink in the study but tried it in the tutorial gave a very positive comment. When asked for one positive comment on ISEE, user25 indicated her preference for "the timestamp on the chat".
6.3.4. Online chat. There were 323 messages sent to the chat room in the entire study. Figure 6 depicts the distribution of messages over the session. The most active user contributed 30 messages and each user sent at least one message. The average number of messages each user sent was 11.5. The number of messages sent to the chat room dropped nearly $50 \%$ in phase III (111 messages) compared to phase II (212). This is natural because the video channel added in phase III would divide part of the cognition time from the devoted text chat.

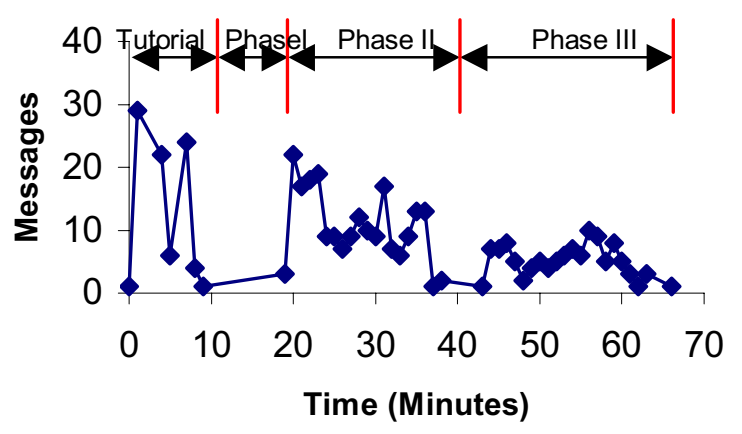

Figure 6. Message Distribution Over Session

When the participants were asked to give one negative comment about the ISEE, to our surprise, the chat room received the most criticism. Some participants (25\%) indicated that too many users in one chat room made the ICR "crowded". As a result, "it was hard to read everything" (user6); "It was difficult to keep up with the chat" (user17); and it was "hard to really isolate a comment I found pertinent" (user27). Only 3.6\% participants think they relied completely on the opinions from the online chat. In addition, two participants believed that there was bias in the text chat contents and one participant (user29) indicated that the anonymity made the comments from others untrustworthy.

From another perspective, many criticisms and comments from the users also suggest the critical role that the text chat played in this experience. As mentioned by one participant in the study, "Through the chat I was able to see other perspectives and able to re-evaluate my opinion of the books" (user1), and "I really liked reading the peer comments. They were very helpful"'(user3). More than half $(53.6 \%)$ of the participants changed their book selection decisions in terms of choosing different books or re-ranking the same selections over the course of the three phases.

\section{Discussion}


In ISEE information components, such as text chat, video, and the shared browser, provide an enriched collaborative learning environment. A comment from user14 illustrates this: "The combination of reviews, chat, and video is helpful to make educated choices." However, cognitive theories suggest that there is a limit to the capacity of an individual's working memory, measured in terms of information chunks. Instructions needed to be carefully designed to reduce the learners' cognitive load [1,21]. With multiple information presentation and communication components being juxtaposed together, it is natural to ask the questions: Will users feel comfortable using ISEE and not feel "overloaded"? How can the system and user interface be optimized to minimize cognitive load?

\subsection{Cognitive load in ISEE}

In the two Yes/No questions on the questionnaire, all the participants indicated that ISEE provided an effective platform to help them complete their tasks and that they had a comfortable experience in using the ISEE system. As one participant (user2) commented: "This is a wonderful learning tool...I have not spotted a fault yet. It's cool stuff'.

In phase III, when all the information channels were available, including reviews, video and text chat, some participants (14.3.\%) felt distracted, especially when the chat comments were not synchronized with the video they currently watched (user10, user12, user15, user23). Some exemplary comments include, "It was difficult to see two windows--say video and chat-- at the same time"(user10); "It is distracting to try reading the chat comments while listening to the video"(user12); “comments don't necessarily match up with timed point in video"(user15). This "distractedness" may explain the drop in chat usage during phase III. As the participants sought to minimize cognitive overload, they focused their attentions on only one information channel. Iignoring other channels in favor of the channel most users deemed the most helpful and informative (video) may be a strategy employed by users to reduce cognitive load. Thus, we can see that overall, subjects were positive about ISEE but that issues of overload and coordination among information channels bear consideration. Clearly, instructors who use such environments should take this into consideration in designing activities for students and should discuss strategies to balance cognitive load as students become familiar with the environment. Likewise, digital libraries that provide client-side tools for users should take these results into account in developing such services.

\subsection{Managing cognitive load}

A desktop user interface provides a flexible environment for people to manage cognitive load by selecting and moving the information components. Even though some participants just accepted the default user interface during the entire session, most of the participants $(82.1 \%)$ rearranged the layout of the user interface to meet their specific needs as they worked. For example, students were observed enlarging the video player, minimizing the SWB, enlarging the ICR, and putting the ICR in the center of the user interface. One user even enlarged the video player to occupy all the available display estate, thus filtering out other information components (Figure 7) for part of the work session. Most students liked having this flexibility. User3 commented, "Having all three items available at once was great; you could select when you needed".

Mayer [14] proposed that students learn better when corresponding information, such as words and pictures, are presented simultaneously. Smartlink is our attempt in ISEE to build quick and direct links between related individual information components. User10 noted: "I liked being able to chat while watching the videos and linking straight to the spot where another user was at when commenting.". This field study demonstrates that such links facilitate the management of multiple information channels and possibly helps people to better manage the cognitive load associated with multiple information channels.

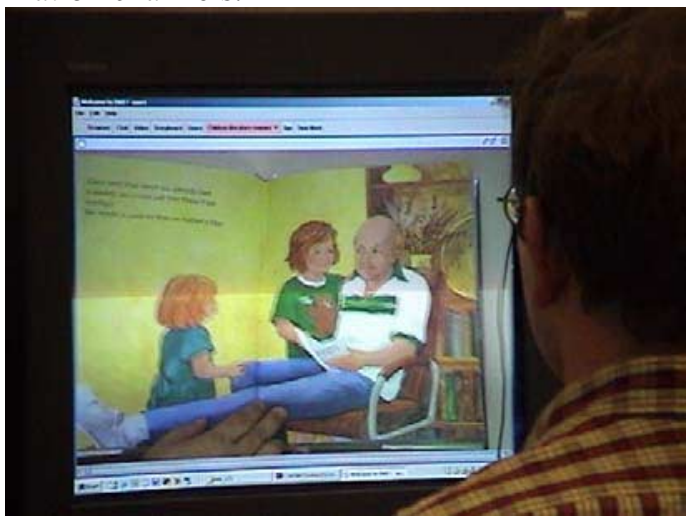

Figure 7: Screenshot of One User's ISEE User Interface

\subsection{Future work}

The ISEE is an evolving distance learning environment and improvements are needed before it can be widely applied in practice. The ICR with smartlink support is a novel feature and was liked by participants in this study. In this version of ISEE, both the instructors and the students viewed the same user interface. A function for the instructor to broadcast a question to the 
entire class is also available but was not used in any of our tests so far.

New features are planned that distinguish the user interface of the instructor from the students. The instructor will be able to survey the students with instant messages. Further, control over the students' video players will be added to the instructor's interface. A multicast video/audio streaming module will be added back in future versions as an option so that fast network users are able to take full advantage of their bandwidth. Audio communication was mentioned as an important communication channel by participants and may be added to the next generation of ISEE. New field studies with heterogeneous network connection speeds are planned to explore the relationship between smartlinks and cognitive load.

\section{Conclusions}

The increasing volume of videos in digital libraries provides an immense resource for distance learning and education. Collaboration and cooperation activities are encouraged by modern learning theories [23]. This study showed how ISEE might be used in a "real life" collaborative setting. The students were engaged in a simulation activity, mimicking the kind of interaction that could support and enhance a common professional task: the selection of materials for a library's collection. Data in the form of student comments shows that the participants actively considered information from multiple sources. An integrated multimedia environment, like that provided by ISEE, discourages passive information use. In a digital library environment, various interactions offered by a rich environment such as ISEE can enhance both the information seeking and information assimilation tasks.

It is a challenge to develop a real-time multimedia collaborative distance-learning environment that coherently integrates video, text chat, and text documents without overloading users' working memory. Based on lightweight communication architectures, ISEE supports synchronizations across four information components: a shared web browser, a interactive chat room, video storyboards, and a video player. Videos can be prepackaged for quick access and navigation functions. Users are able to connect with each other using slower dialup connections.

This classroom field study indicated that the combination of video and text chat is an effective and helpful setting for some decision-making tasks. Smartlinks that are able to quickly present related information from various information channels were introduced in ISEE to minimize the cognitive load in the information-enriched environment. Participants felt comfortable in using the ISEE environment to select children's literature for a library. They reported that video was highly useful in helping them to make their comparisons and selections and although video and chat together increased cognitive load, they were able to use smartlinks to help manage some of this load. These results are promising and will guide continued development and evaluation of ISEE.

\section{Acknowledgements}

Our thanks go to Dr. Barbara Wildurmth for her support in the IRB preparation and to the students who participated in the field study. This work was supported by NSF Grant \#0099638 and the Open Video Project.

\section{References}

[1] Anderson, J. (1983). The Architecture of Cognition. Cambridge, MA: Harvard University Press.

[2] BMRC Lecture Browser: http://bmrc.berkeley.edu/frame/projects/

[3] Brown, B. \& Fortosky,D. (1986). Use of television. In I. Mugridge \& D. Kaufman(Eds.), Distance education in Canada(pp. 260-282). London, England: Croom Helm.

[4] Cadiz, J., Balachandran, A., Sanocki, E., Gupta, A., Grudin, J. and Jancke, G. Distance learning through distributed collaborative video viewing. Proc. CSCW 2000, 135-144.

[5] Chapanis, A., Ochsman, R. B., Parrish, R.N., and Weeks, G.D. (1972). Studies in interactive communication: The effects of four communication modes on the behavior of teams during a cooperative problem solving. Human Factors, 14,487509.

[6] Chen, M. 2001, Design of a Virtual Auditorium. Proceedings of ACM Multimedia 2001,19-28

[7] Deshpande, S.G., and Hwang, J.,2001, A real-time interactive virtual classroom multimedia distance learning system, IEEE Transactions on Multimedia, Vol.3, No. 4, 2001. 432-443

[8] eClass: http://www.cc.gatech.edu/fce/eclass/

[9] Gibbons, J. F., Kincheloe, W.R., and Down, K.S.(1977). Tutored videotape instruction: a new use of electronics media in education. Science. 195:11391146.

[10]Heinich, R., Molenda, M. \& Russell, J. D. (1989). Instructional media and the new technologies of instruction ( $3^{\text {rd }}$ ed.) New York: Macmillan Publishing Company. 
[11] Jancke, G., Grudin, J., and Gupta, A., 2000. Presenting to Local and Remote Audiences: Design and Use of the TELEP System. Proc. CHI 2000, 384391.

[12] Just-In-Time Lecture (JIT): http://www.jitl.cs.cmu.edu/

[13] Maly, K., Abdel-wahab, H., Wild, C., Overstreet, C.M., Gupta, A., Abdel-hamid, A., Ghanem, S., Gonzalez, A., and Zhu, X., 2001, IRI-h, A JavaBased distance education system: architecture and performance. ACM Journal of Educational Resources in Computing, Vol.1, No.1, Spring 2001, Article \#6, 15 pages

[14] Mayer, R. E. 2001, Multimedia Learning. Cambridge University Press.

[15] MediaSite Live: currently available at $\mathrm{http} / / /$ sonicfoundry.com/systems/mslive.asp

[16] Shirmohammadi, S. 2001, Web-based multimedia tools for sharing educational resources, ACM Journal of Educational Resources in Computing, Vol.1, No.1, Spring 2001, Article \#7, 13 pages.

[17] Sipusic, M., Pannoni, R., Smith, R., Dutra, J., Gibbons., J., and Sutherland, W.(1999). Virtual Collaborative learning: A Comparison between Faceto-Face tutored Video Instruction and Distributed
Tutored Video Instruction (DTVI). Sun

Microsystems Laboratories, Inc. TR-99-72.

[18] Smith, R., Sipusic, M., and Pannoni, R. (1999).

Experiments Comparing Face-to-Face with Virtual

Collaborative Learning. Sun Microsystems

Laboratories, Inc. TR-99-0285.

[19] Stanford University's Stanford Online program: http://scpd.stanford.edu/scpd/about/delivery/stanford Online.htm

[20] Stone, H. R.(1990). Economic development and technology transfer: Implications for video-based distance education. In M.G.Moore(Ed.), Contemporary issues in American distance education(pp231-242). Oxford, England: Pergamon.

[21] Sweller, J., Cognitive load during problem solving: Effects on learning, Cognitive Science, 12, 257-285 (1988).

[22] White Pine's CU-SEEME: http://www.cuseeme.com [23] Vygotsky, L.S. (1978). Mind in Society. Cambridge, MA: Harvard University Press.

[24] Yaskin, D. \& Gilfus, S. Introducing the Blackboard 5: learning system. Available online at http://company.blackboard.com/docs/cp/orientation/E nterpriseLearningWhitePaper.pdf 